\title{
Teaching practical astronomy at a professional observatory
}

\begin{abstract}
Summary
In 1988 Southampton University established a new Programme of Study, Physicswith-Astronomy and it was felt to be essential to provide the students with detailed practical experience in observational astronomy. The obvious difficulties of scheduling and successfully executing observational work from the UK led to the establishment of an annual Field Trip to the Observatario del Teide at Izana in Tenerife, Spain. The course allows 12 second-year astronomy students to visit the observatory in Tenerife every Easter vacation for one week.
\end{abstract}

Subject area: Astronomy

\section{Structure \& timetable}

The structure of the main component of this course is built around a six night stay at the observatory run by the Instituto de Astrofisica de Canarias which consists of a collection of day and night time telescopes, together with a suite of experiments studying the cosmic microwave background. It is located at an altitude of $2400 \mathrm{~m}$ about 1 hours drive from La Laguna.

Accommodation and catering is provided on-site, so the astronomical experience is a total one for the students. The course normally starts by arriving around midday allowing a period of familiarisation in day time before work will begin that night. The evening meal occurs around $7 \mathrm{pm}$ and the students are expected to commence work just after sunset i.e. about $8.30 \mathrm{pm}$. Observations will continue, weather permitting, till dawn (around 7am) with a "night lunch" break occurring around lam. Though the first night is normally particularly challenging as people are adjusting their body clocks to a night time schedule, students and staff quickly settle into an effective routine which will last for all 6 nights.

The students are divided up into groups of 3-4 and will spend a whole night working at one of several observational locations. These locations have varied over the years depending upon astronomical circumstances (availability of comets, planets etc). The following have been, and hopefully will continue to be used: 


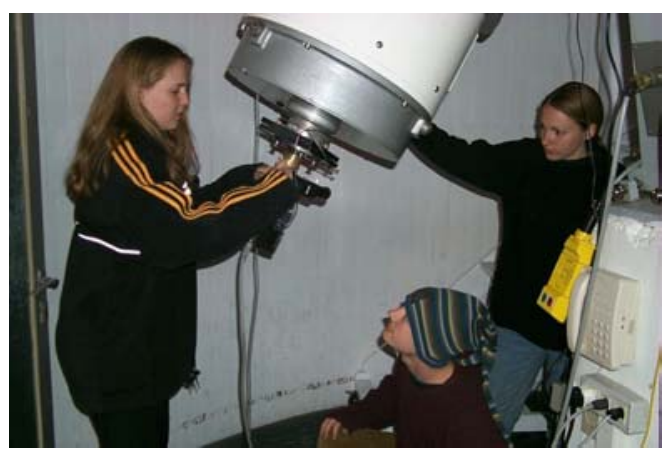

Figure 1 Astronomy students attaching a ST-6 CCD camera to the $0.5 \mathrm{~m}$ Mons telescope

The $0.5 \mathrm{~m}$ Mons telescope has provided the core of our programme upon which we mount a SBIG ST-6 CCD camera with BVRI Johnson filters. This configuration permits observations of both extended objects (e.g. stellar clusters, galaxies \& nebulae). The filters permit quantifiable results to be obtained and this is strongly encouraged in general on this course. By using standard Johnson filters the students are able to obtained Hertzsprung-Russell diagrams of open and globular clusters, colour studies of different types of galaxies and compare their results directly with the published literature

1. The Vacuum Newtonian provides an excellent facility for planetary work due to its exceptionally long focal. The design of this telescope is ideal for detailed studies on Jupiter, Saturn and the moon. The students carry out photographic work using $35 \mathrm{~mm}$ cameras mounted on this system with the objectives of recording Jovian moon rotation, as well as high quality images of all of these systems (to record, for example, the rotation rate of Jupiter by following the Red Spot).

2. A portable Meade LX200 telescope provides a third location for more general familiarisation of the sky and wide-field photographic imaging. Mounting of $35 \mathrm{~mm}$ cameras on the back is straightforward.

3. In addition to night time work, the students are able to spend late afternoons carrying out solar studies using the same LX200 with a Thousand Oaks glass solar filter. The objectives are to photographically record sunspot locations, sunspot development and differential solar motion by taking images over several days and pooling the data from all groups. 


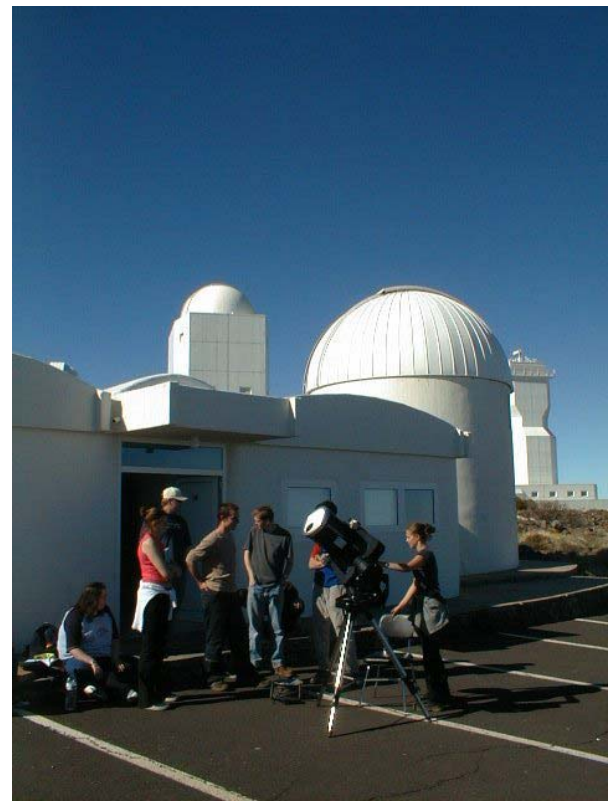

Figure 2 Astronomy students carrying out solar photographic measurements using an LX200 telescope with a solar filter

A photographic darkroom supports many of the above activities. The students are trained to carry out developing and printing of black and white photography. They process their own films and print up their results during the week. In some cases it is essential to develop films almost immediately to provide the necessary feedback on the correct exposure times required. Though this kind of image recording is rapidly diminishing in popularity at all levels, it nonetheless provides a rigorous and scientifically demanding test of their skills.

On return to Southampton the students are set two objectives to encourage the analysis and interpretation of their data under more considered circumstances. These objectives are, firstly, to produce an A1 poster for presentation at an in-house conference, and secondly to write an essay discussing some other aspect of their work.

\section{Learning outcomes}

After participating on this course it is expected that our students should be able to:

1. work in small teams with each member having a specific responsibility

2. employ practical skills in operating complex equipment often under difficult weather conditions (e.g. cold temperatures and brisk winds)

3. operate a telescope and find their way around the sky in celestial co-ordinates

4. process data using image processing programmes on PCs.

5. present the results of their work verbally, illustratively and in writing.

\section{Feedback}

This type of intensive course is excellent for obtaining feedback on student progress and learning. Contact between the staff and students are extensive and prolonged, and 
there are many opportunities to observe student progress and to spot difficulties in the learning process. It is also often very straightforward to adapt the process in real time so as to bypass the cause of any serious difficulties. The details of the course have evolved over its lifetime, but there have been many examples of major improvements being made because of the close observation of the students at work.

More formally, we issue all students a standard questionnaire at the end of their course to obtain more balanced reflective feedback. The results of these questionnaires have always revealed an exceptionally high rating for this course and considerable student satisfaction with what they have achieved. They find the course to be extremely demanding, but they enjoy the unusual challenges presented by a programme that is so different to all their other traditional laboratory or lecture based programmes.

\title{
Conclusions
}

This course has proved an exceptionally successful way of teaching practical astronomical observation techniques to astronomy undergraduates. It has stimulated many of them to pursue the subject in much greater depth than they had previously planned, including inspiring many to study for $\mathrm{PhDs}$ in this area. Variations on this course are being actively explored, or have already been established, by several other universities.

\section{Acknowledgements}

We gratefully acknowledge the considerable assistance provided by many staff who have helped with this course over the years, in particular Dr Ismael Perez Fournon of the University of La Laguna and the Instituto de Astrofisica de Canarias.

\author{
Author(s) \\ Dr Malcolm J Coe \\ Dept of Physics \& Astronomy, University of Southampton
}

\section{Contact details}

Dr Malcolm J Coe

Dept of Physics \& Astronomy, University of Southampton, SO17 1BJ, UK.

mjcoe@soton.ac.uk 